\title{
GINCANA PARA O ENSINO DE IMUNIZAÇÃO AOS ACADÊMICOS DE ENFERMAGEM: RELATO DE EXPERIÊNCIA
}

\author{
GAMES FOR TEACHING IMMUNIZATION TO NURSING STUDENTS: EXPERIENCE REPORT
}

\section{GINCANA PARA LA ENSEÑANZA DE INMUNIZACIÓN A LOS ACADÉMICOS DE ENFERMERÍA: RELATO DE LA EXPERIENCIA}

\author{
Bruna Hinnah Borges Martins de Freitas ${ }^{1}$
}

\begin{abstract}
RESUMO
Objetivo: Descrever a experiência na construção e aplicação de uma gincana para o ensino de imunização aos acadêmicos de Enfermagem. Método: Trata-se de um relato de experiência de uma gincana que foi construída com base em cinco jogos adaptados ao conteúdo de imunização: dramatização, paródia, tabuleiro, palavra cruzada e, verdadeiro ou falso. Esse conteúdo é abordado na disciplina de Enfermagem em Saúde da Criança e do Adolescente de uma Universidade Pública do Centro-Oeste Brasileiro. Resultados: Todos foram aplicados para acadêmicos de Enfermagem, durante duas aulas, com duração de oito horas. Os acadêmicos são divididos em quatro grupos e vence o grupo que obtiver maior pontuação na gincana. A maior dificuldade encontrada foi a falta de preparo por parte dos alunos para a gincana, com pouco estudo prévio sobre a temática, consequência de os estudantes não estarem habituados a trabalhar em grupo de forma participativa, assumindo o protagonismo do seu aprendizado. Entretanto, considera-se que os jogos foram recursos positivos para a resolução de problemas e reflexão sobre o conteúdo teórico. Conclusão: Conclui-se que os jogos podem ser utilizados no ensino quando adaptados ao conteúdo desejado, pois permitem o aprofundamento teórico de maneira lúdica e prazerosa aos estudantes.
\end{abstract}

Descritores: Educação em enfermagem; Estudantes de enfermagem; Jogos e brinquedos; Material de ensino.

\section{ABSTRACT}

Objective: To describe an experience of construction and application of a game section for teaching immunization to nursing students. Method: This is an experience report of a game section that was built based on five games adapted to the immunization content: dramatization, parody, board, crossword and true or false. This content is addressed in the Child and Adolescent Health Nursing course at a Public University in the Midwest of Brazil. Results: The games were applied to nursing students during two classes, lasting eight hours. The students were divided in four groups and the group with the highest score in the contest was the winner of the competition. The greatest difficulty encountered by the students was their lack of preparation for the competition, with little previous study about the topics, which was a consequence of the lack of the habit among students of working in groups in a participatory way, assuming a protagonist learning behavior. However, it is considered that the game section was a positive resource for solving problems and reflecting on the theoretical content. Conclusion: It is concluded that games can be used in teaching when adapted to the desired content, since they allow theoretical deepening in a playful and pleasurable way for students.

Descriptors: Nursing Education; Nursing Students; Games and toys; Teaching materials.

\section{RESUMEN}

Objetivo: Describir la experiencia en la construcción y aplicación de una gincana para enseñar inmunización a estudiantes de enfermería. Método: Este es un informe de la experiencia de una gincana que se construyó con base en cinco juegos adaptados al contenido de inmunización: dramatización, parodia, tablero, crucigrama y verdadero o falso. Este contenido se aborda en la disciplina de Enfermería en Salud Infantil y Adolescente en una Universidad Pública del Medio Oeste de Brasil. Resultados: Todos se aplicaron a estudiantes de enfermería durante dos clases, con una duración de ocho horas. Los académicos se dividen en cuatro grupos y gana el grupo con la puntuación más alta en el concurso. La mayor dificultad encontrada fue la falta de preparación por parte de los estudiantes para la competencia, con poco estudio previo sobre el tema, una consecuencia de que los estudiantes no estaban acostumbrados a trabajar en grupos de manera participativa, asumiendo el protagonismo de su aprendizaje. Sin embargo, se considera que los juegos fueron recursos positivos para resolver problemas y reflexionar sobre el contenido teórico. Conclusión: Se concluye que los juegos se pueden usar en la enseñanza cuando se adaptan al contenido deseado, ya que permiten la profundización teórica de una manera lúdica y placentera para los estudiantes.

Descriptores: Educación en Enfermería; Estudiantes de Enfermería; Juego e Implementos de Juego; Materiales de Enseñanza.

${ }^{1}$ Mestre em Enfermagem. Docente da Faculdade de Enfermagem, Universidade Federal de Mato Grosso, Cuiabá, Mato Grosso, Brasil.

\section{Como citar este artigo:}

Freitas BHBM. Gincana teórica para o ensino de imunizações aos acadêmicos de enfermagem: Relato de Experiência . Revista de Enfermagem do Centro Oeste Mineiro. 2020;10:e2959. [Access ]; Available in: DOI: 


\section{INTRODUÇÃO}

Atualmente, o grande desafio no ensino superior é a ruptura dos modelos de ensino tradicionais de profissionais de saúde vigentes até então, que se baseiam, sobretudo, numa postura de transmissão de informações, na qual os alunos assumem um papel passivo no processo de ensino-aprendizagem ${ }^{(1)}$.

Especificamente, a formação de enfermeiros ainda ocorre, em sua maioria, por meio da ministração de aulas expositivas, sem a participação efetiva dos alunos na construção do conhecimento $^{(2)}$. Para mudar esse paradigma, é necessária uma relação dialógica entre educador e educando, possibilitando o aprendizado mútuo, por meio de um processo emancipatório ${ }^{(3)}$. Sendo que, uma das maneiras de atingir esse propósito é a aprendizagem mediada.

A aprendizagem mediada é uma forma de interação que produz atitudes e competências básicas para uma aprendizagem efetiva, oportunizando uma dinâmica de estruturação do conhecimento. $\mathrm{O}$ mediador se interpõe entre $\mathrm{O}$ aprendiz e o mundo dos estímulos, facultando a interpretação e a significação. Um dos mediadores do processo de ensino-aprendizagem são os jogos, que se figuram como método alternativo que geram estímulos aos estudantes $^{(4)}$.

Os jogos educativos são àqueles que dispõem de um desígnio didático manifesto e conseguem ser adotados ou adaptados para aperfeiçoar, sustentar ou viabilizar os processos de aprendizagem. Esses jogos contêm regras e permitem o entretenimento, carecendo, como qualquer outro recurso didático e metodológico, possuir objetivos definidos e coerência nas estratégias utilizadas ${ }^{(5)}$.

Estes têm sido utilizados como recursos educacionais, em várias áreas do conhecimento, como: Medicina, Enfermagem, Pedagogia, Psicologia e Letras. Os relatos são de que os jogos possuem uma característica motivadora, por mobilizar os jogadores, promovendo uma oportunidade para a discussão. Ao passo que, no contexto do jogo, à medida que o jogador aprende o conteúdo educativo que compõe o jogo, são concebidas, além dos reforçadores arbitrários, consequências inerentes à própria aprendizagem $^{(6)}$.

Considera-se que os jogos não são apenas um recurso interativo e motivante, mas também uma tecnologia capaz de gerar aprendizagem, promover diálogo, facilitar a abordagem de temas, esclarecer dúvidas e debater situações cotidianas $^{(6)}$. Isso só é possível quando o material assente a partir do contexto do aprendiz, de suas experiências e vivências, para que, a partir delas, ele possa construir o seu conhecimento.

Além disso, a participação em jogos permite ao acadêmico conhecer materiais educacionais que podem auxiliar o ensino mais participativo, tecnologia essencial nas práticas educativas do enfermeiro. Porquanto, materiais de ensino dinamizam as atividades de educação em saúde. Esse papel de educador que o enfermeiro possui é fundamental para o empoderamento de indivíduos, famílias e comunidades com vistas à melhora nas condições de saúde e qualidade de vida ${ }^{(7)}$.

$O$ processo de formação do enfermeiro deve possibilitar que o indivíduo se torne críticoreflexivo e político, preparando-o para ser agente transformador da sociedade em que atua. Mudanças na prática de formação pedagógica corroboram para esse $\mathrm{fim}^{(3)}$. Na enfermagem, a utilização de jogos no ensino é reconhecida por promover interação e envolvimento entre os participantes, estimular interesse em um determinado tópico e prover elementos para mudança de atitude ${ }^{(8)}$.

Por isso, experiências pedagógicas voltadas à mudança de processos representam um movimento inovador na reelaboração da aprendizagem significativa para os alunos, favorecendo a rupturas de paradigmas educacionais tradicionais e legitimando movimentos mais amplos de mudança ${ }^{(1)}$. Nesse sentido, o intuito deste estudo foi descrever a experiência na construção e aplicação de uma gincana para o ensino de imunização aos acadêmicos de Enfermagem.

\section{MÉTODO}

Trata-se de um relato de experiência de uma docente, na construção e aplicação de uma gincana para o ensino de imunização aos acadêmicos de Enfermagem de uma Universidade Pública do Centro-Oeste brasileiro.

O conteúdo de imunização é contemplado na disciplina de Enfermagem em Saúde da Criança e do Adolescente, partindo do pressuposto que a formação do enfermeiro tem por objetivo dotar o profissional dos conhecimentos requeridos para o exercício da competência de atenção à saúde com habilidades para desenvolver ações de prevenção de doenças e promoção da saúde, tanto em nível individual 
quanto coletivo. Sabe-se que a imunização é uma das estratégias mais eficaz na redução da morbimortalidade infantil e nos custos com saúde ${ }^{(8)}$.

A disciplina em questão é ofertada no sétimo semestre, no respectivo curso de graduação, sendo disponibilizadas oito horas para se trabalhar o conteúdo programático, que envolve todo o Plano Nacional de Imunização, orientações de Normas e Procedimentos para Vacinação e Vigilância Epidemiológica de Eventos Adversos Pós-Vacinação e, o cuidado em enfermagem na imunização.

A construção da gincana teórica, para o ensino da imunização, ocorreu em abril e maio de 2017, com o intuito de estabelecer a aprendizagem mediada por jogos, possibilitando um aprendizado mais significativo, quando comparado à aula expositiva dialogada anteriormente ofertada, na perspectiva da docente. A aplicação da mesma ocorre desde então, em todos os semestres nas duas aulas destinadas a esse conteúdo, com os alunos matriculados na disciplina.

\section{RESULTADOS E DISCUSSÃO}

\section{Construção da gincana teórica para o ensino de imunização}

A construção da gincana teórica baseou-se no Plano Nacional de Imunização, Manual de Normas e Procedimentos para Vacinação e Manual de Vigilância Epidemiológica de Eventos Adversos Pós-Vacinação do Ministério da Saúde e, outros materiais acerca do cuidado de enfermagem na imunização.

A mesma foi elaborada contendo cinco jogos didáticos, como pode ser visualizado no Quadro 1 e pensada na composição de quatro grupos (A, B, C e D), definidos por sorteio. Compreendem-se os jogos como um desafio que garante o dinamismo entre pessoas de interesse comuns e tem por objetivo a recreação e a construção do conhecimento entre os pares, a qual possibilita reflexão e associação com a prática profissional $^{(4)}$. Além disso, a resolução de problemas e o pensamento crítico são objetivos educacionais que podem ser atingidos com a utilização de jogos ${ }^{(9)}$.

Quadro 1- Jogo, objetivo, conhecimento trabalhado e tempo de aplicação na gincana de ensino sobre imunização aos acadêmicos de Enfermagem. Cuiabá, MT, Brasil, 2020.

\begin{tabular}{|c|c|c|c|}
\hline Jogo & Objetivo & Conhecimento trabalhado & $\begin{array}{l}\text { Tempo de } \\
\text { aplicação }\end{array}$ \\
\hline Dramatização & $\begin{array}{l}\text { Grupo A: Demonstrar os fundamentos dos } \\
\text { imunobiológicos, por meio de uma aula a } \\
\text { acadêmicos de enfermagem. } \\
\text { Grupo B: Demonstrar o início e o término do } \\
\text { trabalho diário na sala de vacina. } \\
\text { Grupo C: Demonstrar como deve ocorrer o } \\
\text { acolhimento e a vacinação de uma criança. } \\
\text { Grupo D: Demonstrar as orientações necessárias } \\
\text { quanto aos Eventos Adversos Pós-Vacinação (EAPV) } \\
\text { comuns a vários imunobiológicos e condutas. }\end{array}$ & $\begin{array}{l}\text {-Fundamentos de imunologia; } \\
\text {-Organização e funcionamento da sala } \\
\text { de vacina; } \\
\text {-Equipamentos e insumos da sala de } \\
\text { vacina; } \\
\text {-Acolhimento; } \\
\text {-Brinquedo Terapêutico; } \\
\text {-Contraindicações aos imunobiológicos; } \\
\text {-Administração dos imunobiológicos; } \\
\text {-Intervenção para o alívio da dor; } \\
\text {-EAPV comuns a vários } \\
\text { imunobiológicos; } \\
\text {-Condutas frente aos EAPV comuns a } \\
\text { vários imunobiológicos. }\end{array}$ & 3 horas \\
\hline Paródia & Apresentar uma paródia sobre o calendário vacinal. & $\begin{array}{l}\text {-Calendário vacinal; } \\
\text {-Ludicidade; } \\
\text {-Criatividade. }\end{array}$ & 1 hora \\
\hline Tabuleiro & $\begin{array}{l}\text { Compreender o conceito de vacina, situações de } \\
\text { adiamento, indicação, esquema, idade máxima e } \\
\text { mínima, dose e via de administração das vacinas. }\end{array}$ & $\begin{array}{l}\text {-Conceito de vacina; } \\
\text {-Situações de adiamento de uma } \\
\text { vacina; } \\
\text {-Indicação, esquema, idade máxima e } \\
\text { mínima, dose e via de administração } \\
\text { das vacinas. }\end{array}$ & 2 horas \\
\hline $\begin{array}{l}\text { Palavra } \\
\text { cruzada }\end{array}$ & $\begin{array}{l}\text { Refletir acerca do Plano Nacional de Imunização e } \\
\text { das Normas e Procedimentos para Vacinação. }\end{array}$ & $\begin{array}{l}\text {-Plano Nacional de Imunização; } \\
\text {-Normas e procedimentos gerais para } \\
\text { Vacinação. }\end{array}$ & 1 hora \\
\hline $\begin{array}{l}\text { Verdadeiro ou } \\
\text { Falso }\end{array}$ & $\begin{array}{l}\text { Discutir sobre a Vigilância Epidemiológica de } \\
\text { Eventos Adversos Pós-Vacinação. }\end{array}$ & $\begin{array}{l}\text {-Vigilância Epidemiológica de Eventos } \\
\text { Adversos Pós-Vacinação. }\end{array}$ & 1 hora \\
\hline
\end{tabular}


A dramatização, a ser elaborada previamente pelos alunos, deve ser apresentada em 30 minutos por cada grupo, tendo 15 minutos no término para a discussão entre os pares, com participação da docente nos esclarecimentos quanto aos pontos positivos e negativos da encenação, conforme as recomendações do Ministério da Saúde e dos demais materiais fornecidos. Os grupos que cumprirem com os objetivos traçados somam cinco pontos na gincana.

Na sequência, a apresentação da paródia de forma criativa e lúdica culmina em cinco pontos. No término das apresentações, o calendário vacinal é apresentado aos alunos na lousa pelo docente e as dúvidas são esclarecidas.

No segundo dia de aula, os demais jogos são aplicados. Primeiramente, o jogo do tabuleiro, no qual a cada jogada um representante do grupo deve lançar o dado e andar as casas, conforme o número sorteado. No jogo, existem 38 casas, sendo 15 de casas numéricas, 15 de paradas com questões, duas de bônus, cinco de ônus e a chegada (Figura 1).

As paradas correspondem a perguntas acerca do conceito de vacina, situações de adiamento, indicação, esquema, idade máxima e mínima, dose e via de administração das vacinas. Sendo que, se o jogador acertar a resposta ele andará algumas casas e, caso ele erre, retornará algumas casas, conforme orientação do roteiro do jogo. O bônus corresponde a atitudes positivas descritas na casa como: "Parabéns, você aplicou o brinquedo terapêutico. Ande duas casas" e o ônus como: "Você se esqueceu de higienizar as mãos, volte ao local de partida".
Essas colocações ressaltam as atitudes assertivas ou não que o profissional de enfermagem pode ter na sala de vacinação e promove a reflexão acerca da prática.

Esse jogo foi impresso em lona, nas medidas $100 \mathrm{~cm}$ de altura por $80 \mathrm{~cm}$ de largura, necessitando de um dado e quatro peças coloridas, sendo uma para cada grupo de competidores. O grupo que atingir o local de chegada primeiro somará 15 pontos na competição, o que chegar por segundo 10 pontos e, por terceiro, cinco pontos. No término, a temática do jogo deve ser recapitulada com a finalidade de abordar as principais dúvidas dos alunos.

O quarto jogo corresponde à palavra cruzada. Cada grupo tem o impresso em mãos para responderem juntos (Figura 2), seguindo as orientações do Quadro 2. O grupo que finalizar primeiro com $100 \%$ de acertos terá 10 pontos na gincana. Sequencialmente, são revistas todas as afirmativas que geraram dúvidas aos alunos.

Para finalizar, o quinto jogo trata-se do jogo do verdadeiro ou falso, elaborado no aplicativo Kahoot! ${ }^{\circledR}$, composto por trinta e duas afirmações sobre a Vigilância Epidemiológica de Eventos Adversos Pós-Vacinação e cada grupo tem que responder conforme o seu julgamento, se verdadeira ou falsa. A equipe que tiver mais acertos somará 10 pontos na competição. Também são esclarecidas todas as dúvidas dos alunos após o término do jogo. Vence o grupo com maior pontuação.

Figura 1- Jogo do tabuleiro para gincana de ensino sobre imunização aos acadêmicos de Enfermagem. Cuiabá, MT, Brasil, 2020.

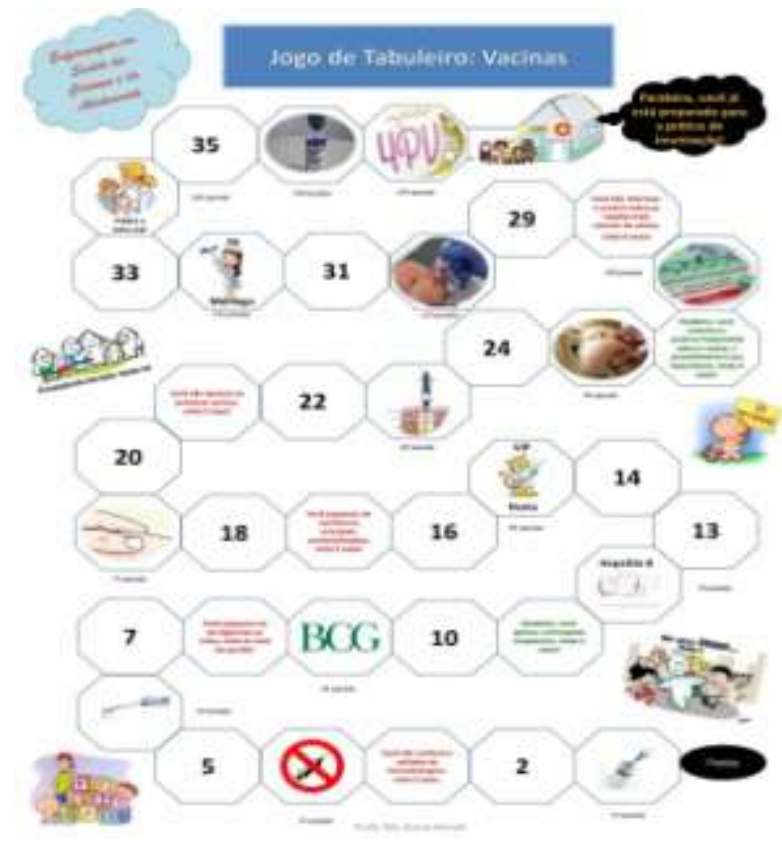


Figura 2- Palavra cruzada para gincana de ensino sobre imunização aos acadêmicos de Enfermagem. Cuiabá, MT, Brasil, 2020.

\section{PALAVRA CRUZADA: VACINAS}

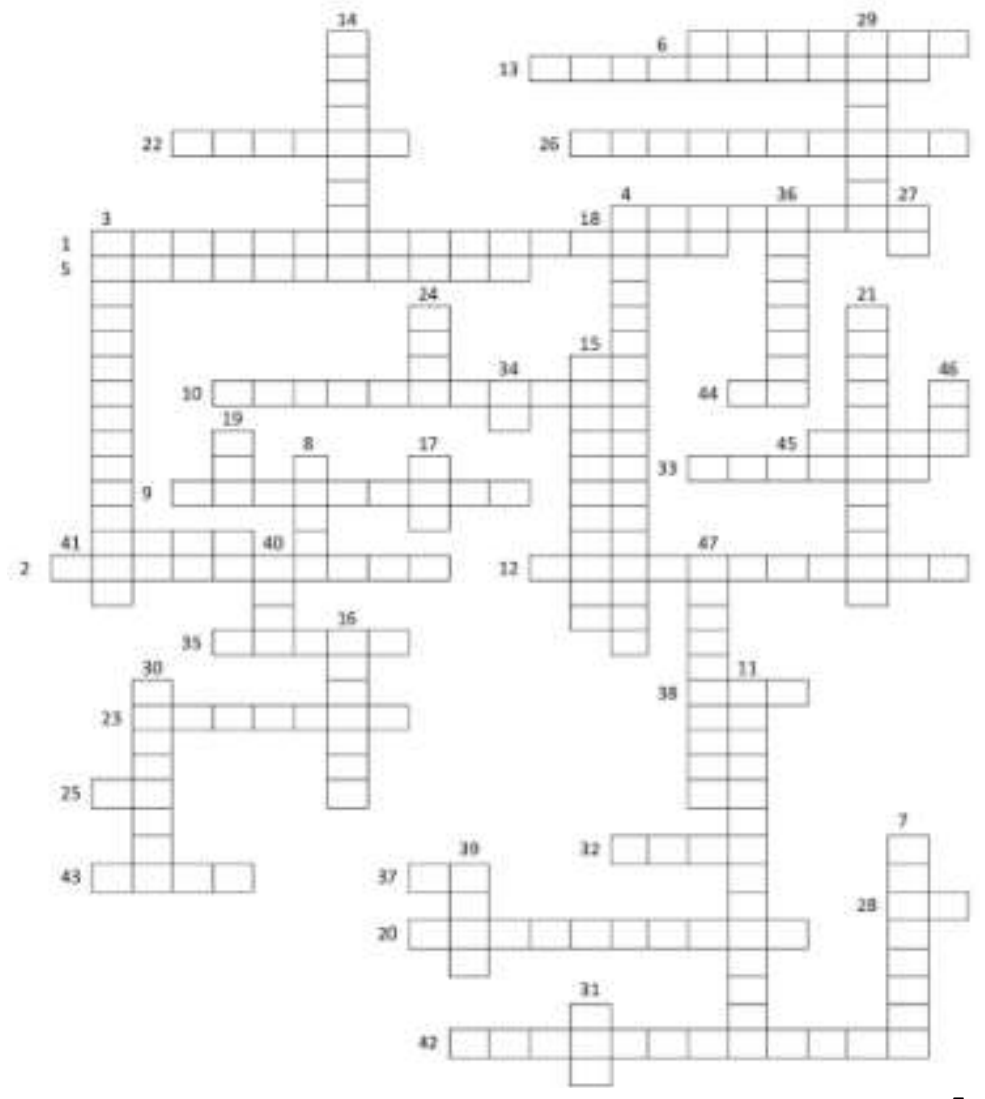

Fonte: Elaborado pela autora.

Quadro2- Orientação para preenchimento da palavra cruzada para gincana de ensino sobre imunização aos acadêmicos de Enfermagem. Cuiabá, MT, Brasil, 2020.

\footnotetext{
1- O Programa Nacional de Imunização tem como missão o controle, a erradicação e a eliminação de quais doenças?

2- É a forma de definição das vacinas ofertadas na rotina dos serviços de saúde.

3- É um dos imunobiológicos disponibilizados pelo Programa Nacional de Imunização.

4- A forma em que o sistema imune atua mais lentamente, com produção de anticorpos específicos.

5- Local onde as células da resposta imunológica são desenvolvidas.

6- Imunidade mediada pelos linfócitos B.

7- Resposta em que a primeira classe de imunoglobulina a ser produzida é a IgM e, posteriormente, a IgG.

8- Imunidade obtida pela estimulação da resposta imunológica com a produção de anticorpos específicos.

9- Não devem receber vacinas vivas, pois existe a possibilidade de os antígenos vivos atenuados causar alguma alteração, como, por exemplo, malformação.

10- Alguns indivíduos podem apresentar esse tipo de reação a alguns componentes dos imunobiológicos.

11. Esses pacientes devem ser avaliados, caso a caso, para administração adequada de imunobiológicos.

12. Seu uso como profilático é recomendado apenas em crianças com história pessoal e familiar de convulsão e para aqueles que tenham apresentado hipertermia ou choro incontrolável após dose anterior da vacina DTP.

13. Substâncias presentes na composição de algumas vacinas e que aumentam a resposta imune dos produtos que contêm microrganismos inativados e seus componentes.

14. Responsável técnico pela sala de vacina.

15. Atitude de inclusão na sala de vacina.

16. Processo de escolha, seleção ou classificação no qual os usuários são submetidos a fim de determinar aqueles que possuem prioridade.

17. Vacina contra difteria, tétano e coqueluche (sigla).

18. O seu gerenciamento deve estar em conformidade com a definição estabelecida na RDC ANVISA no306 de 2004.

19. Local onde os resíduos infectantes devem ser encaminhados em saco branco leitoso nas caixas coletoras (sigla).

20. Sistema utilizado pelo PNI, que tem o objetivo de assegurar que os imunobiológicos sejam mantidos em condições adequadas.
} 
21. Processo logístico que envolve o recebimento, armazenamento, distribuição e transporte dos imunobiológicos.

22. Local onde ficam os imunobiológicos na sala de vacina.

23. Insumos importantes para a conservação dos imunobiológicos nas caixas térmicas.

24. Via de administração da vacina VOP.

25. Via de administração da BCG (sigla).

26. Não deve ser feito no local de administração da vacina BCG.

27. Via de administração em que a vacina é introduzida na hipoderme (sigla).

28. Via de administração em que o volume máximo é de até $5 \mathrm{ml}$ (sigla).

29. Deve ser feito na caderneta de vacinação da criança.

30. Taxa que mede a adesão do usuário ao programa de imunização.

31. Âmbito de disponibilidade dos imunobiológicos gratuitamente (sigla).

32. Local de encaminhamento dos usuários que fizeram transplante de medula óssea (sigla).

33. Deve ocorrer com a vacinação de indivíduos em uso de droga imunossupressora de corticoide.

34. Doença prevenida pela vacina BCG (sigla).

35. Vacina que possui o esquema de três doses, aos 2, 4 e 6 meses (sigla).

36. Uma das doenças preveníveis pela DTP.

37. Vacina que possui reforço a cada 10 anos (sigla).

38. Vacina inativada indicada para prevenir contra a poliomielite (sigla).

39. Número de doses da vacina contra poliomielite.

40. Número de meses em que a criança deve fazer o primeiro reforço da vacina contra pneumonia.

41. Número de meses em que a criança faz a vacina contra febre amarela.

42. Vacina indicada para prevenir contra infecções invasivas como sepse, meningite, e otite média aguda.

43. Local ideal de se colocar a criança com o corpo reclinado para a administração da vacina rotavírus.

44. Vacina conhecida por ser indicada para viajantes para áreas de risco para a doença (sigla).

45. Pode acontecer após a vacinação e deve ser investigado (sigla).

46. Vacina que deve ser administrada especificamente na adolescência (sigla).

47. Vacina que protege contra o sarampo, caxumba, rubéola e varicela.

Fonte: Elaborado pela Autora.

\section{Aplicação da gincana aos acadêmicos de Enfermagem}

Precedente à gincana, os alunos matriculados na disciplina Enfermagem em Saúde da Criança e do Adolescente são instruídos quanto à gincana, no início do período letivo, e os materiais didáticos que a norteiam são distribuídos aos alunos para estudo prévio. A comunicação entre professor-aluno ocorre com cerca de quinze dias de antecedência, a fim de oportunizar o preparo dos mesmos para a atividade programada. Assim, no dia das aulas de Imunização, a gincana é desenvolvida com os acadêmicos de Enfermagem.

O preparo dos alunos se refere ao estudo do conteúdo programático da aula, a elaboração e o ensaio da dramatização e da paródia, componentes da gincana educativa.

Percebe-se dedicação por parte dos alunos quanto à dramatização e a paródia, contudo, verifica-se certa dificuldade na condução dos demais jogos, pois são poucos os alunos que estudam previamente o conteúdo conforme o recomendado. Isso, por vezes, compromete a discussão entre os pares, devido ao pouco conhecimento acerca do conteúdo, uma vez que o preparo teórico por parte dos estudantes é fundamental para o desenvolvimento de tais atividades.
Durante a dramatização, é possível identificar tanto pontos assertivos quanto não assertivos nas encenações, de modo que a discussão entre os pares e a docente seja necessária no fomento de questionamentos que originem reflexão, e exposição por parte dos alunos das mudanças necessárias para garantir uma cena coerente com o que é preconizado pelo Ministério da Saúde e demais materiais norteadores da gincana.

Quanto à paródia, geralmente, todos os grupos a expõe de modo criativo e lúdico, demonstrando estudo do calendário vacinal de imunização para a composição da paródia.

Os demais jogos, frequentemente, são permeados de dúvidas e incertezas. As situações de aprendizagem geradas pelos jogos auxiliam os participantes a emitirem respostas apropriadas ou não em relação ao conteúdo que o jogo se proponha, produzindo consequências que possam ser identificadas e reforçadas. Quando não apropriada, abre-se para a discussão entre os pares e, na sequência, a docente assume a condução teórica acerca dos questionamentos levantados.

Segundo os próprios estudantes, a falta de preparo prévio por parte deles é um empecilho que prejudica a gincana. Entretanto, justifica-se que os mesmos não estejam habituados a trabalhar em grupo, de forma participativa e 
dialógica, cujo método implica na responsabilização por parte dos estudantes no preparo para a aula, por meio da leitura dos materiais disponibilizados pela docente. Tal atitude é fundamental para prover a participação ativa dos estudantes durante o processo de construção de conhecimento.

De acordo com pesquisadores ${ }^{(2)}$, geralmente os docentes se utilizam de pedagogias tradicionais, ministrando aulas expositivas e sem a participação efetiva dos estudantes, sendo eles os protagonistas do processo ensino-aprendizagem e não os estudantes. O que condiciona a tais dificuldades encontradas.

É essencial que o ensino em saúde incorpore os avanços pedagógicos das metodologias ativas de ensino-aprendizagem, estabelecendo novas diretrizes para a integração dos saberes e o rompimento com a tradicional dicotomia entre ensino básico e formação clínica $^{(2)}$.

A gincana possibilita aos acadêmicos a resolução de problemas, a compreensão dos jogos e dos conteúdos específicos trabalhados. Verifica-se que, as regras e as dicas fornecidas, durante a atividade, favorecem o aprendizado dos alunos, à medida que eles levantam hipóteses, analisem e interpretem a resolução dos problemas e discutem com o grupo para a emissão de uma resposta. Assim, se desenvolve a reflexão crítica necessária para a atuação em imunização.

A docente, na condução da gincana, assume o papel de provocadora do raciocínio dos alunos, procura gerar desequilíbrios cognitivos em relação ao conteúdo e, possibilita interações ativas com o conhecimento e aprendizagem significativa $^{(1)}$.

\section{Avaliação do material}

Observa-se que a implementação desse material didático-pedagógico facilita e dinamiza o processo de ensino-aprendizagem no tocante à temática em questão, pois os jogos se configuram como um recurso valioso para a resolução de problemas e reflexão sobre o conteúdo teórico. Este possibilita um ambiente promotor de discussões e da construção de conhecimentos ${ }^{(5-6)}$.

Nota-se que este material é eficiente no ensino e aprendizagem, pois proporciona o conhecimento da temática trabalhada pelo estímulo ao entusiasmo do aprendiz e por seu maior envolvimento. Isso ocorre, em razão da promoção da comunicação e manifestação espontâneas entre os pares, sendo a docente a articuladora das discussões.

Constata-se que, os jogos presentes na gincana representam momentos de aprendizagem significativa, relaxada, motivada, descontraída, os quais ressaltam o conhecimento e o aprendizado como algo mais amplo do que o contexto acadêmico permite. Eles integram aos jogos às experiências e conhecimentos pessoais que cada jogador traz consigo. Com essa finalidade, a gincana parece auxiliar todo o processo de aprendizagem e de desenvolvimento cognitivo, afetivo e emocional ${ }^{(4)}$.

A competitividade é intrínseca ao ser humano e o desejo de vencer revela um maior interesse por parte dos aprendizes, na interiorização do conhecimento, a partir da associação de ideias ${ }^{(9)}$. Esse aspecto se conecta com a abordagem da aprendizagem problematizadora, por criar oportunidades de síntese do conhecimento.

Os alunos expressaram-se, positivamente, a essa estratégia de ensino, ressaltando a dinamicidade e motivação durante a aula. Contudo, este estudo limita-se por ser um relato de experiência da docente, sendo necessários estudos posteriores para avaliar a percepção dos estudantes acerca do material.

\section{CONCLUSÃO}

Conclui-se que essa experiência permitiu reconhecer a gincana de imunização, por meio de jogos, como um recurso que favorece o processo de ensino-aprendizagem, assegurando a participação ativa dos alunos na construção de seus próprios conhecimentos. Assim, compreende-se que essa estratégia deva ser aprimorada e utilizada por docentes a fim de permitir o aprofundamento teórico sobre imunização de maneira lúdica e prazerosa.

\section{REFERÊNCIAS}

1- Siqueira MCG, Leopardi MT. O processo ensino-aprendizagem na formação de trabalhadores do SUS: Reflexões a partir da experiência da ETSUS. Trab Educ Saúde 2016;14(1):119-36. DOI: 10.1590/1981-7746sip00094

2- Chavaglia SRR, Barbosa MH, Santos AS, Duarte RD, Contim D, Ohl RIB. Estratégias didáticas identificadas junto a graduandos de Enfermagem. Cogitare Enferm. 2018;3 (23):1-9. DOI: 10.5380/ce.v23i3.53876 
3- Backes DS, Zamberlan C, Siqueira HCH, Backes MTS, Sousa FGM, Lomba MLLF. Quality nursing education: A complex and multidimensional phenomenon. Texto Contexto-Enferm. 2018;27(3):1-7. DOI: 10.1590/0104070720180004580016

4- Ramos DK, Lorenset CC, Petri G. Jogos educacionais: Contribuições da neurociência à aprendizagem. Rev X 2016;2:1-17. DOI: 10.5380/rvx.v2i1.2016.46530

5- Dondi C, Moretti M. A methodological proposal for learning games selection and quality assessment. Br J Educ Technol. 2007;38(3):50212. DOI: 10.1111/j.1467-8535.2007.00713.x 6- Panosso MG, Souza SR, Haydu VB. Características atribuídas a jogos educativos: Uma interpretação analítico-comportamental. Psicol Esc Educ. 2015;19(2):233-41. DOI: 10.1590/2175-3539/2015/0192821

7- Silva JP, Gonçalves MFC, Andrade LS, Monteiro EMLM, Silva MAI. Health promotion in primary education: perceptions of bachelor's degree with a teaching diploma in nursing students. Rev Gaúcha Enferm. 2018;39:e20170237. DOI: https://doi.org/10.1590/19831447.2018.2017-0237

8- Gurgel SS, Taveira GP, Matias EO, Pinheiro PNC, Vieira NFC, Lima FET. Educational games: Didactic resources utilized at teaching health education classes. Rev Min Enferm. 2017;21: 1-6. DOI: 10.5935/1415-2762.20170026

9- Gurgel SS, Taveira GP, Matias EO, Pinheiro PNC, Vieira NFC, Lima FET. Jogos educativos: Recursos didáticos utilizados na monitoria de Educação em saúde. Rev min enferm. 2017 [citado em 2017 dez 13]; 21:e1016. Available in: http://www.reme.org.br/artigo/detalhes/1152

Nota: Este relato não obteve financiamento de nenhuma agência de fomento.

Recebido em: $11 / 06 / 2018$

Aprovado em: 09/04/2020

Endereço de Correspondência:

Faculdade de Enfermagem

Universidade Federal de Mato Grosso

Av. Fernando Corrêa da Costa, n2367- Bairro Boa

Esperança, CEP: 78060-900

E-mail: bruhinnah@gmail.com 